\title{
Revisiting Silica with ReaxFF: Towards Improved Predictions of Glass Structure and Properties via Reactive Molecular Dynamics
}

\author{
Yingtian Yu, ${ }^{1} \mathrm{Bu}$ Wang, ${ }^{1}$ Mengyi Wang, ${ }^{1}$ Gaurav Sant, ${ }^{2,3}$ and Mathieu \\ Bauchy ${ }^{1}$ \\ ${ }^{1}$ Physics of AmoRphous and Inorganic Solids Laboratory (PARISlab), Department of Civil and \\ Environmental Engineering, University of California, Los Angeles, California, 90095, USA \\ ${ }^{2}$ Laboratory for the Chemistry of Construction Materials $\left(L C^{2}\right)$, Department of Civil and Environmental \\ Engineering, University of California, Los Angeles, California, 90095, USA \\ ${ }^{3}$ California Nanosystems Institute (CNSI), University of California, Los Angeles, California, 90095, USA
}

Reactive potentials, like ReaxFF, are becoming increasingly popular and are expected to bridge the gap between $a b$ initio and classical molecular dynamics simulations. Yet, their applicability to glassy materials remains poorly understood. Here, by simulating a silica glass with both ReaxFF and a conventional classical potential, we critically assess the ability of reactive potentials to offer an accuracy equivalent or superior to that of classical potentials in describing the structure and properties of glassy silicates. We show that ReaxFF produces a realistic disordered structure, both at the short- and medium-range order. Interestingly, ReaxFF offers significant improvements with respect to classical potentials in describing the elastic properties of the glass and the dynamics of the supercooled liquid. Overall, while retaining a reasonable computational cost, ReaxFF appears as a promising potential to model the structure and properties silicate glasses and, in contrast to classical potential, could be used to assess their surface reactivity.

Keywords: Amorphous silica, Molecular dynamics, ReaxFF

\section{INTRODUCTION}

$\mathrm{ReaxFF}^{1}$ is a reactive force field based on a dynamic bond order, which is determined on the fly with respect to the instantaneous bond distance. The concept of bond distance to bond order relationship was first introduced by Tersoff $^{2}$ and, later, implemented within the carbon environment by Brennan ${ }^{3}$. ReaxFF is based on this concept and formulates the potentials as functions of bond orders, while the bonds are allowed to dynamically form and dissociate. As such, in contrast to conventional classical inter-atomic potentials, the framework of ReaxFF allows chemical reactions to be properly described. In addition, the parameters of ReaxFF are fitted and optimized against an extensive database of quantum mechanics calculations and experimental data. All together, these features enable

Corresponding author.

E-mail addresses: bauchy@ucla.edu (M. Bauchy)

(C) 2016. This manuscript version is made available under the Elsevier user license http://www.elsevier.com/open-access/userlicense/1.0/ 
the simulations of large atomic systems, in complex chemical environments, with a computational cost comparable to that typically required by classical force fields.

The span of successful applications of ReaxFF includes a broad range of carbon-based systems ${ }^{4-6}$, silicon and silicon oxide (crystal structures) systems ${ }^{1,7}$, titania ${ }^{8,9}$, aluminum and aluminum oxide systems ${ }^{10}$, etc. ReaxFF has also been shown to accurately describe the hydration of systems such as calcium-silicate-hydrates ${ }^{11-13}$, and the mechanical properties of various materials ${ }^{14-17}$. In addition to crystalline solids, ReaxFF has been used to study the glassy silica-water interface ${ }^{18}$ and organosilicate glasses ${ }^{19}$. In fact, the application of ReaxFF to glasses is especially attractive, as their structure, which, in contrast to crystals, is not a priori known, remains poorly understood. However, non-crystalline structures are usually not taken into account during the development of potentials. As such, it remains unclear whether ReaxFF, primarily parameterized versus crystalline networks, can produce realistic glasses. In addition, simulations of glasses are also particularly challenging, as modeled glasses are typically produced by quenching a system from its liquid state, thereby mimicking the experimental procedure used to form glasses. Hence, such a methodology requires the used potential to offer a realistic description of the solid as well as the liquid and super-cooled liquid states. As such, there is a need to assess in details the ability of reactive potentials like ReaxFF to predict realistic structures for glassy materials.

Here, we focus on glassy silica, which serves as a structural basis for various multi-component silicate glasses of both industrial and geological interests ${ }^{20}$. By following a consistent procedure to form the glass, the outcome of the ReaxFF potential is compared to that of a conventional classical force field. Overall, ReaxFF is shown to offer a realistic description of silica glass. The present paper is organized as follows. First, the details of the simulations are presented in Sec. 2. A structural, mechanical, and dynamical characterization of the glasses and supercooled liquids is then presented in Sec. 3. Eventually, these results are discussed in Sec. 4 and summarized in Sec. 5.

\section{METHODOLOGY}

\subsection{ReaxFF force field}

ReaxFF partitions the total energy of an atomic system $E_{\text {system }}$ into several energy components ${ }^{21}$ :

$$
E_{\mathrm{system}}=E_{\mathrm{bond}}+E_{\mathrm{over}}+E_{\mathrm{under}}+E_{\mathrm{lp}}+E_{\mathrm{val}}+E_{\mathrm{pen}}+E_{\mathrm{torsion}}+E_{\mathrm{conj}}+E_{\mathrm{vdWaals}}+E_{\text {Coulomb }}
$$

where the components include, following the order above, bond energy, over-coordination energy, undercoordination energy, lone electron pairs energy, valence angle energy, penalty energy, torsion energy, conjugation energy, van der Waals energy and Coulomb potential energy. The prominent feature separating ReaxFF from 
conventional classical force fields is that the covalent interactions (e.g., bond energy, valence angle energy, torsion energy, etc.) are determined from a dynamic bond order, which is updated continuously from the instantaneous interatomic distances during a simulation ${ }^{1}$. In particular, ReaxFF is able to formulate single, double and triple bonds $\left(\mathrm{BO}^{\sigma}, \mathrm{BO}^{\pi}, \mathrm{BO}^{\pi \pi}\right)$, each having a distinct dependence on the inter-atomic distance and on different dissociation energies. The bond energy $E_{\mathrm{bond}}$ can be described by the following form:

$$
E_{\mathrm{bond}}=-D_{\mathrm{e}}^{\sigma} \mathrm{BO}_{i j}^{\sigma} \exp \left[p_{\mathrm{be}, 1}\left(1-\left(\mathrm{BO}_{i j}^{\sigma}\right)^{p_{\mathrm{be}, 2}}\right)\right]-D_{e}^{\pi} \mathrm{BO}_{i j}^{\pi}-D_{e}^{\pi \pi} \mathrm{BO}_{i j}^{\pi \pi}
$$

where $D$ and $p$ are the bond energy parameters, which can be found in Ref. 1. This allows ReaxFF to capture the dynamics of bond forming and breaking processes, which is required to simulate chemical reactions. The form of the function used for the bond order in ReaxFF also ensures that the covalent interactions disappear smoothly upon bond dissociation, after which only the non-bonded and Coulomb interactions play a role.

In addition, an implemented charge equilibration $(\mathrm{QEq})$ method $^{22,23}$ allows the atom charges to be assigned dynamically. As such, the charge of an individual atom depends on its environment (e.g., its coordination number). This is a very attractive feature for glassy materials, as the coordination number of the atoms (like $\mathrm{B}$ or $\mathrm{Al}$ ) can show some variability 24,25 . On the contrary, conventional classical force fields maintain constantly the full or partial charges for each element. The QEq method also provides the convenient feature of having finite atom sizes, which shields Coulomb interactions at short distances and resolves the difficulties encountered by excluding the Coulomb interaction for dynamically bonded atoms. Similar short distance shielding is applied to van der Waals term as well 21.

ReaxFF also includes energy terms to account for various bond natures. The over-coordination is compensated by a penalty energy $\left(E_{\text {over }}\right.$, while $E_{\text {under }}$ accounts for $\square$ electrons that are found between the bonded undercoordinated atoms. Furthermore, an additional energy penalty term $E_{\mathrm{pen}}$ is superimposed to ensure the stability of the system when two double bonds are shared by atoms in a valence angle. The heat of formation for non-conjugated closed shell molecules is reproduced through the $E_{\text {conj }}$ term. To determine all the parametric values used in ReaxFF, the parameters set is optimized against a large database containing data from quantum chemical calculations of both molecular and crystal systems, as well as from experiments. More details of these energy terms can be found in Ref. 1.

\subsection{Glass preparation}


All simulations in this study are performed using the LAMMPS package ${ }^{26}$. The ReaxFF potential is implemented through the user-reaxc package. The outcome of ReaxFF is compared to that of classical molecular dynamics (MD), relying on the well-established BKS potential ${ }^{27}$, which is probably the most commonly used for silica. Various potentials have been proposed (see, e.g., Ref. 28), each having its own advantages. Nonetheless, the BKS potential remains the most commonly applied potential, thanks to its satisfactory performance while retaining a simple formulation. Although we keep in mind that some superior force fields are available, such as polarizable interatomic force fields ${ }^{29}$, they usually have more complex forms and, therefore, come with higher computational cost. On the other hand, despite its simplicity, the BKS potential has been shown to produce a realistic bulk structure for silica ${ }^{30,31}$. Here, we use a modified version of the original BKS potential, with a short-range cutoff $5.5 \AA$ and long range Coulomb interaction cutoff $10.0 \AA^{32}$.

Three methods are tested in this study. (1) A glass is formed via quenching a liquid from $4000 \mathrm{~K}$ to $300 \mathrm{~K}$, fully using ReaxFF (ReaxFF method). (2) A glass is formed via quenching a liquid from $4000 \mathrm{~K}$ to $300 \mathrm{~K}$, fully using the BKS potential (classical method). (3) A glass is first formed via quenching a liquid from $4000 \mathrm{~K}$ to $300 \mathrm{~K}$ using the BKS potential, then equilibrated at $300 \mathrm{~K}$ with ReaxFF (hybrid method). Here, the hybrid method is tested as a potential option to avoid the higher computational cost of ReaxFF with respect to usual classical MD during the cooling phase.

More specifically, $\mathrm{SiO}_{2}$ units (4536 atoms) are first randomly distributed inside a cubic box, by ensuring no unfavorable overlap between the atoms. Then, we ensure the loss of the memory of the initial configuration by melting the system at $4000 \mathrm{~K}$ for $100 \mathrm{ps}$. The silica glasses are then prepared by linearly reducing the temperature of the system, from $4000 \mathrm{~K}$ to $300 \mathrm{~K}$. The formed glass is subsequently equilibrated at $300 \mathrm{~K}$ for an additional $1 \mathrm{~ns}$, although no additional relaxation is observed at this point. The NPT ensemble, ensuring a zero pressure, is applied throughout the process. In the case of the hybrid method, a glass is first produced with the BKS potential by cooling the liquid from $4000 \mathrm{~K}$ to $300 \mathrm{~K}$, before being subsequently relaxed with the ReaxFF potential at $300 \mathrm{~K}$ for $1 \mathrm{~ns}$. This length of time was found to be sufficient to ensure the equilibration of both the energy and the volume of the system. Due to the high bonding energy of $\mathrm{SiO}_{2}$, we do not observe any changes in the connectivity of the network, that is, no bond is broken or created during the relaxation process at $300 \mathrm{~K}$. Note that a consistent cooling rate of 1 $\mathrm{K} / \mathrm{ps}$ is used for all the three methods. Previous studies have shown that such a cooling rate is sufficient to obtain a realistic glass structure ${ }^{32-34}$, even though it is considerably higher than those used in experiments. Note that cooling 
rates as high as $5 \sim 10 \mathrm{~K} / \mathrm{ps}$ are commonly used in MD simulations, due to the computational cost of the technique. The effect of the cooling rate has recently been carefully evaluated ${ }^{35}$ and, although the density and thermal expansion appear to be affected, most of the structural features of the glass remain unchanged.

\section{RESULTS}

\subsection{Medium-range order}

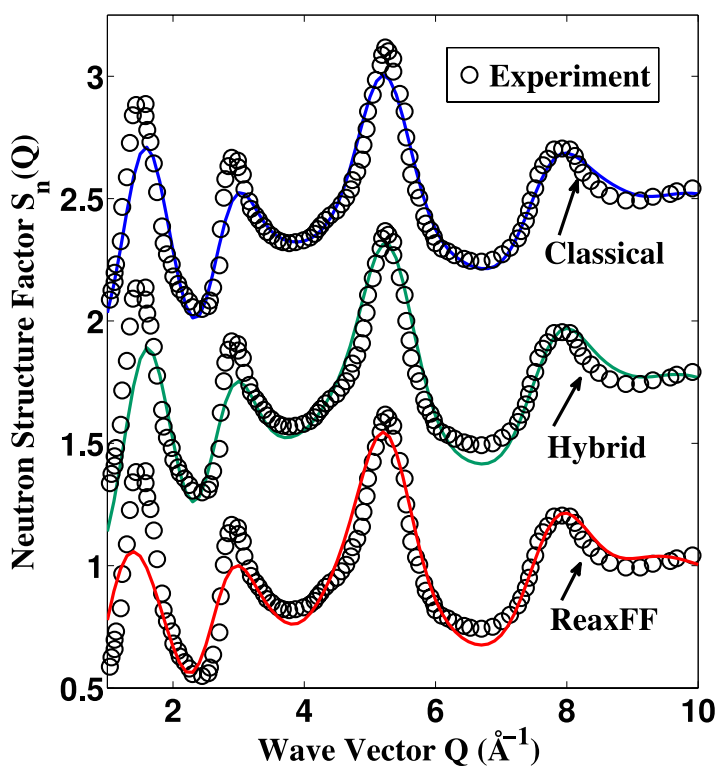

FIG. 1. Computed neutron structure factors of the simulated glasses, as prepared with the ReaxFF, hybrid and classical methods (lines). The results are compared each with the same data from diffraction experiments ${ }^{36}$.

To assess the simulated structure of silica glass, we first compute the neutron structure factor $S_{\mathrm{N}}(Q)$ by calculating the Fourier-transform of the partial pair distribution functions (PDF) $g_{\mathrm{ij}}(r)$. Note that a Lorch-type window function is used to avoid potential spurious ripples at low $Q$ caused by the finite cutoff used during the Fourier-transform ${ }^{37}$. The obtained neutron structure factors are shown in Figure 1, and compared with results from diffraction experiments ${ }^{38}$.

As shown in Figure 1, we observe a very good overall agreement in the high $Q$ range for all the three methods, which suggests a well-reproduced short-range structure. In the low $Q$ range, however, some discrepancies are observed around $1.5 \AA^{-1}$, at the vicinity of the first sharp diffraction peak (FSDP), which suggests that these three methods predict slightly different medium-range orders. Specifically, the BKS potential presents a shift of the FSDP to larger $Q$ values with respect to what is obtained experimentally, whereas the ReaxFF method predicts a slightly 
smaller value. We also note that the structure obtained with the hybrid method is similar to that of ReaxFF at large $Q$, whereas in the low $Q$ region, the FSDP remains reminiscent of that predicted by the BKS potential.

TABLE I. Computed densities of the simulated glasses, as prepared with the three methods. The results are compared with the experimental density.

\begin{tabular}{lcccc}
\hline & Classical & Hybrid & ReaxFF & Experiment $^{\text {a }}$ \\
\hline Density $\left(\mathrm{g} / \mathrm{cm}^{3}\right)$ & 2.22 & 2.34 & 2.18 & 2.20 \\
& & & \\
\hline \hline
\end{tabular}

In fact, these discrepancies are manifested in the density of the formed glasses, which are tabulated in Table I. Indeed, a strong correlation between the position of the FSDP $\left(Q_{\mathrm{FSDP}}\right)$ and the density has been noted in previous studies, i.e., larger $Q_{\text {FSDP }}$ implies a smaller distance of structural repetition and, hence, higher density, and vice versa $^{40}$. Such a trend is in accordance with what is observed in the present study, i.e., the density predicted by the ReaxFF method is slightly lower than the experimental value, while the classical method offers a slightly higher prediction. The levels of deviation of these two methods are on the same level, i.e., about $0.02 \mathrm{~g} / \mathrm{cm}^{3}$. We note that the hybrid method, on the other hand, produces a larger deviation of $0.14 \mathrm{~g} / \mathrm{cm}^{3}$. Although the level of agreement is similar for the ReaxFF and BKS potentials, it is worthwhile to point out that the BKS potential used in the study has been specifically parameterized to reproduce the density of glassy silica and, for instance, would not predict a correct density for quartz with the same cutoff. On the contrary, ReaxFF is expected to be transferable to a large number of compounds, in various chemical environments. Hence the fact that the ReaxFF method yields the same level of agreement as compared to BKS is encouraging.

\subsection{Short-range order}

Although it contains the same level of information as the structure factor, the total correlation function (TCF) emphasizes the short-range order. As such, both of them should be compared to experiments to assess the quality of a potential. The TCFs, as calculated for the glasses obtained by the three considered methods, are shown in Figure 2 . It is worth noting that the diffraction instruments used in experiments have a finite scattering wave vector $\left(Q_{\text {max }}\right)$. Hence, for meaningful comparisons, we broaden the calculated TCFs using the method described by Wright ${ }^{41}$. 


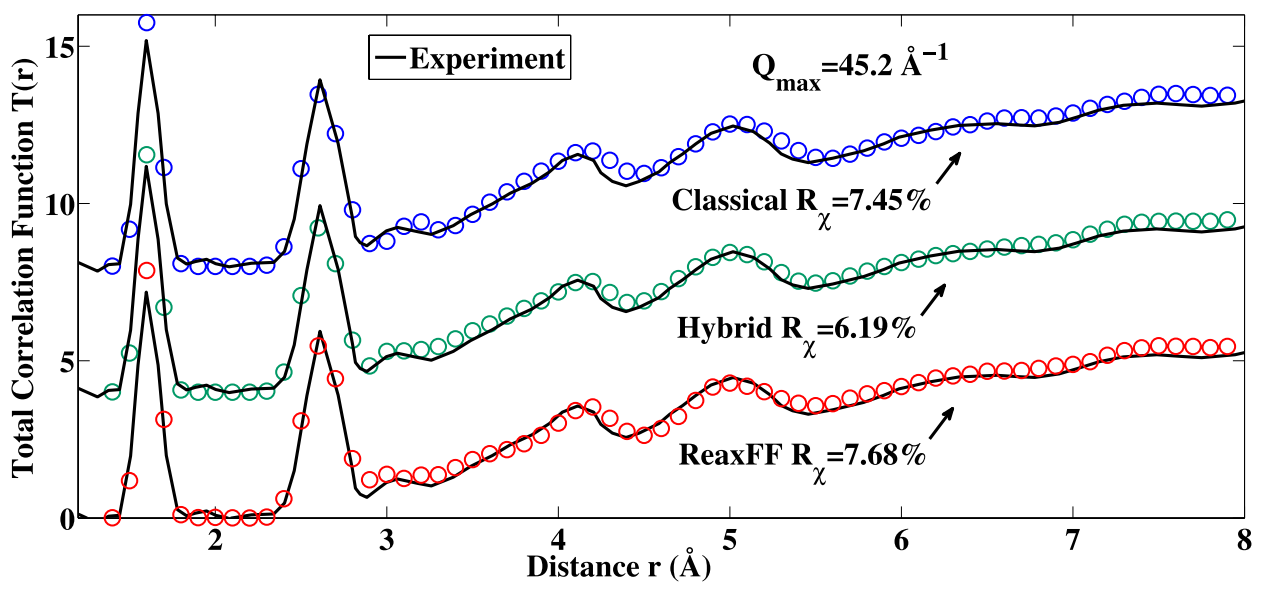

FIG. 2. Computed total correlation functions of the simulated silica glasses, as obtained by the three methods (circles). The results are compared with the same experimental data (solid lines), obtained by neutron diffraction ${ }^{36}$. For each method, the calculated $\boldsymbol{R}_{\chi}$ factors are shown.

To assess the level of agreement between the computed and experimental TCFs, we adopt Wright's method and calculate the $R_{\chi}$ factor between $1 \AA$ and $8 \AA$, which is given by the following equation:

$$
R_{\chi}=\left[\frac{\sum_{i=1}^{n}\left(g(r)-g_{\mathrm{ref}}(r)\right)^{2}}{\sum_{i=1}^{n}\left(g_{\mathrm{ref}}(r)\right)^{2}}\right]
$$

where $g_{\text {ref }}(r)$ is the experimental TCF and $n$ is the total number of points for the TCF. Considering that a $R_{\chi}$ factor of less than $10 \%$ is typically considered to represent a good agreement, we note that all the three methods provide a realistic overall structure, which manifests by $R_{\chi}$ factors being between $6 \%$ and $8 \%$. However, when averaged on all pairs of atoms, the TCFs and structure factors usually appear to be largely unaffected by the details of the potentials ${ }^{25}$. A more detailed analysis of the environment of each element is therefore required.

TABLE II. Computed average $\mathrm{Si}-\mathrm{O}$ bond lengths for the three methods, compared with available experimental values.

Classical Hybrid $\quad$ ReaxFF $\quad$ Experiment

$\begin{array}{cccc}\text { Average } \mathrm{Si}-\mathrm{O} \text { Bond } & 1.613 & 1.615 & 1.608^{\mathrm{a}} / 1.610^{\mathrm{b}} \\ \text { Length }(\AA) & 1.616 & & \end{array}$

${ }^{a}$ Experimental $\mathrm{Si}-\mathrm{O}$ bond length is from Ref. ${ }^{36}$

${ }^{\mathrm{b}}$ Experimental $\mathrm{Si}-\mathrm{O}$ bond length is from Ref. ${ }^{42}$. 
In order to gain more insight into the short-range structure, the partial pair distribution functions (PDFs) are calculated for the $\mathrm{Si}-\mathrm{O}, \mathrm{O}-\mathrm{O}$ and $\mathrm{Si}-\mathrm{Si}$ pairs. The first peak of the partial PDFs, as shown in Figures 3-5, corresponds to the first coordination shell of each element, and allows us to calculate the average inter-atomic bond distances. We note that, for both the $\mathrm{Si}-\mathrm{O}$ and $\mathrm{O}-\mathrm{O}$ PDFs (Figure 3 and Figure 4), no significant difference is observed among the three methods. Indeed, all the three methods predict an average bond length that is in excellent agreement with experiments (see Table II).

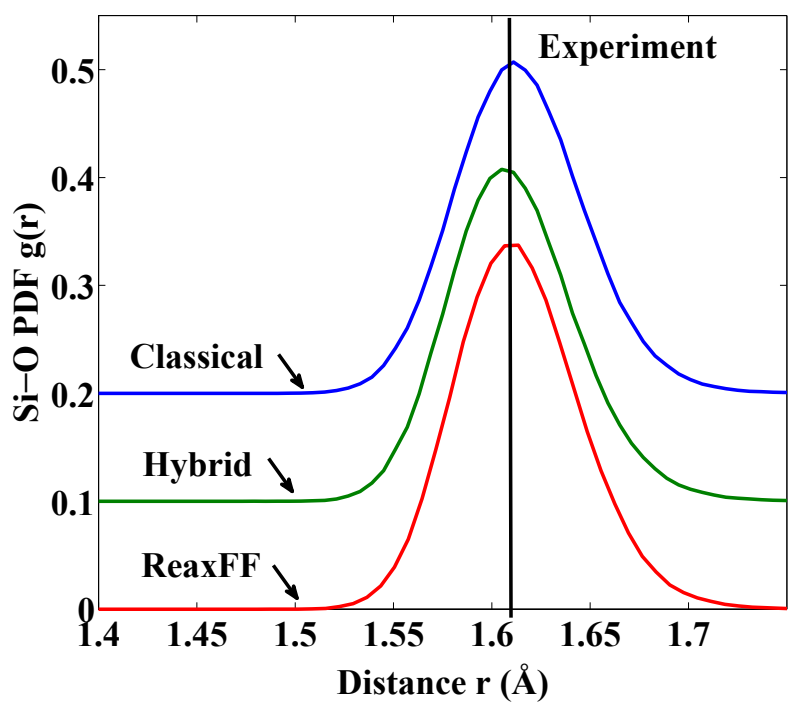

FIG. 3. Computed $\mathrm{Si}-\mathrm{O}$ pair distribution functions, as obtained by the three methods. Available experimental values of the average $\mathrm{Si}-\mathrm{O}$ bond length are represented by solid vertical lines ${ }^{36},{ }^{42}$. Note that, here, the spread of the experimental values is too small to be visible.

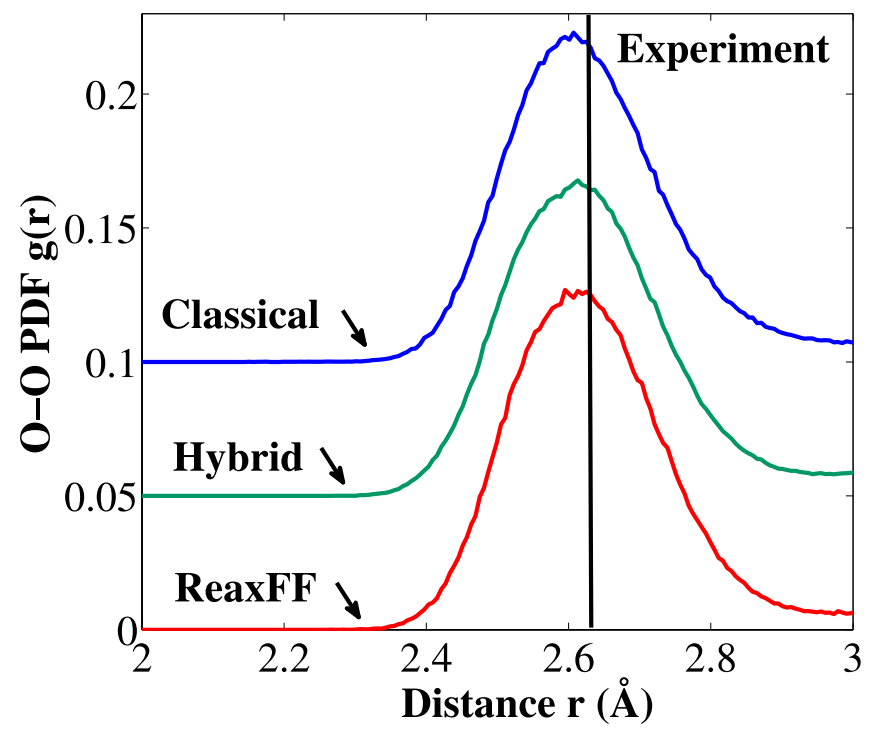

FIG. 4. Computed O-O pair distribution functions, as obtained by the three methods. The experimental value of the average $\mathrm{O}-\mathrm{O}$ bond length is represented by a solid vertical line ${ }^{36}$. 
The coordination number of each species are calculated by directly enumerating the number of neighbors of each atom, with a distance cutoff defined as the first minimum after the main peak of the PDFs. The results are shown in Table III. Overall, we find a good agreement with experimental observations, as all methods predict a realistic tetrahedral environment for $\mathrm{Si}$ atoms, with a coordination number of around $4^{39}$.

TABLE III. Computed coordination numbers of Si atoms, compared with the experimental value.

Classical Hybrid ReaxFF $\quad$ Experiment $^{\mathrm{a}}$

Si Coordination

4.00

3.99

3.99

3.95

Number

${ }^{\mathrm{a}}$ Experimental coordination number is from Ref. ${ }^{39}$.

On the contrary, a noticeable difference among the three methods is observed for the Si-Si PDF. As shown in Figure 5, the three methods predict significantly different shapes and average values for the first peak of the PDF. First, in agreement with previous studies ${ }^{31}$, the BKS potential (classical method) predicts a largely symmetric distribution, centered at $3.11 \AA$. In contrast, both the ReaxFF and the hybrid methods show an asymmetric distribution, centered at lower distances. We note, however, that the hybrid method feature a more pronounced shoulder around $3.13 \AA$, reminiscent of the distribution obtained by the classical method. Since the three methods predict a similar $\mathrm{Si}-\mathrm{O}$ bond length, the present difference observed in the average $\mathrm{Si}-\mathrm{Si}$ distance obviously arises from differences in the $\mathrm{Si}-\mathrm{O}-\mathrm{Si}$ (inter-tetrahedral) bond angle, as discussed in Sec. 3.3. 


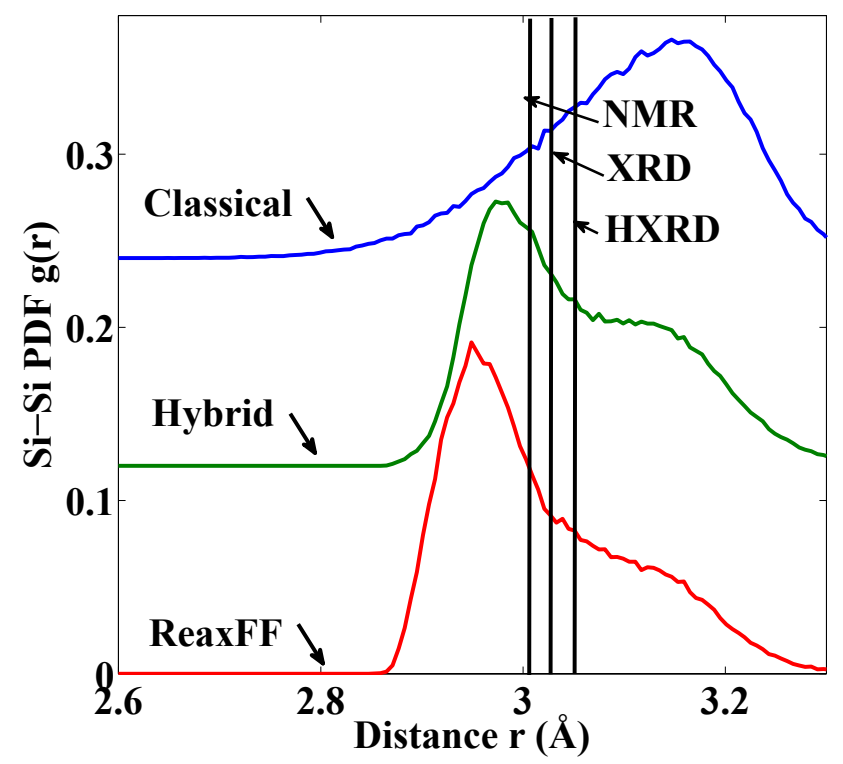

FIG. 5. Computed $\mathrm{Si}-\mathrm{Si}$ pair distribution functions, as obtained with the three methods. The vertical lines indicate available experimental data of the average $\mathrm{Si}-\mathrm{Si}$ bond distance, calculated from the experimental $\mathrm{Si}-\mathrm{O}-\mathrm{Si}$ average angles ${ }^{43-45}$ and the $\mathrm{Si}-\mathrm{O}$ distances $^{36}$.

\subsection{Bond angle distributions}

As expected, the $\mathrm{Si}-\mathrm{O}-\mathrm{Si}$ bond angle distributions (BADs), as shown in Figure 6, also show significantly different shapes and average values. In agreement with previous studies ${ }^{31}$, the BKS classical potential predicts a symmetrical distribution, centered on $152^{\circ}$. In contrast, the ReaxFF potential predicts an asymmetrical distribution, with an average value shifted to lower angle. The hybrid method, once again, retains some features from both the classical and ReaxFF methods, which manifests by an asymmetric distribution featuring a pronounced shoulder reminiscent from the peak predicted by the classical method. Overall, based on the average value of the angle, we observe a reasonable agreement with the available experimental data for ReaxFF. We also note that the distribution produced by the classical method has a full width at half maximum (FWHM) similar to that obtained from standard X-ray diffraction (XRD) (see Table IV), but significantly larger than that obtained by nuclear magnetic resonance (NMR) spectroscopy and high energy XRD (HXRD). However, since the broad FWHM obtained from standard $\mathrm{XRD}$ is likely to be largely affected by the instrument (e.g., X-ray source) being used, the $\mathrm{Si-O}-\mathrm{Si} \mathrm{BAD}$ predicted by the BKS potential appears unrealistically wide. This is likely due to the fact that the BKS potential does not feature any three-body energy terms to constraint the angles. On the other hand, the ReaxFF potential predicts a more realistic narrower distribution, which can potentially offer a better description of the $\mathrm{SiO}_{2}$ network geometry. We also note that experiments suggest the existence of an asymmetrical distribution ${ }^{46}$, which arises from the $\mathrm{Si}-\mathrm{Si}$ 
steric repulsion ${ }^{47}$. However, it is important to recognize that experimental data with higher resolution would be helpful for any further evaluation.

Due to the limited extent of experimental data, the outcome of $a b$ initio simulations could represent an attractive point of reference. As such, the $\mathrm{Si}-\mathrm{O}-\mathrm{Si} \mathrm{BAD}$ obtained from $a b$ initio simulation ${ }^{48}$ is also included for comparison in Figure 6. We note that the obtained average value of the BAD $\left(141^{\circ}\right)$ shows a better agreement with the one predicted by ReaxFF, rather than with the one predicted by BKS. However, the distribution obtained by ab initio simulations shows a lower degree of asymmetry as compared to that predicted by ReaxFF. Nevetherless, it should be kept in mind that $a b$ initio simulation comes their own limitations, which makes it challenging to use their outcome as absolute references. Indeed, the very limited size of the system (72 atoms) and the extremely high cooling rate (on the order of $100 \mathrm{~K} / \mathrm{ps}$ ) used in the ab initio simulation presented here could greatly affect the shape of the $\mathrm{Si}-\mathrm{O}-\mathrm{Si}$ BAD. In fact, a recent study focusing on the role of the cooling rate ${ }^{35}$ indeed showed that the $\mathrm{Si}-\mathrm{O}-\mathrm{Si} \mathrm{BAD}$ is the structural feature that is the most sensitive to the used cooling rate, and that a slower cooling rate result in the appearance of a higher degree of asymmetry. As such, no solid conclusions can be drawn here by comparing the outcome of ReaxFF and BKS with that of ab initio simulations.

TABLE IV. Computed mean bond angle and full width at half-maximum (FWHM) of the $\mathrm{Si}-\mathrm{O}-\mathrm{Si}$ bond angle distributions obtained with the three methods compared with experimental values from NMR, HXRD and XRD are listed for comparison.

Classical Hybrid $\quad$ ReaxFF NMR $^{\mathrm{a}} \quad \mathrm{HXRD}^{\mathrm{b}} \quad \mathrm{XRD}^{\mathrm{c}}$

\begin{tabular}{|c|c|c|c|c|c|c|}
\hline $\begin{array}{c}\text { Mean Bond } \\
\text { Angle }\end{array}$ & $152^{\circ}$ & $145^{\circ}$ & $140^{\circ}$ & $142^{\circ}$ & $147^{\circ}$ & $144^{\circ}$ \\
\hline FWHM & $35^{\circ}$ & $15^{\circ}$ & $14^{\circ}$ & $26^{\circ}$ & $17^{\circ}$ & $38^{\circ}$ \\
\hline
\end{tabular}

The $\mathrm{O}-\mathrm{Si}-\mathrm{O}$ (intra-tetrahedral) $\mathrm{BAD}$ is also computed and shown in Figure 7. As expected, all the three methods produce a similar average peak value, at about $109^{\circ}$, characteristic of the tetrahedral environment of the $\mathrm{Si}$ atoms. 


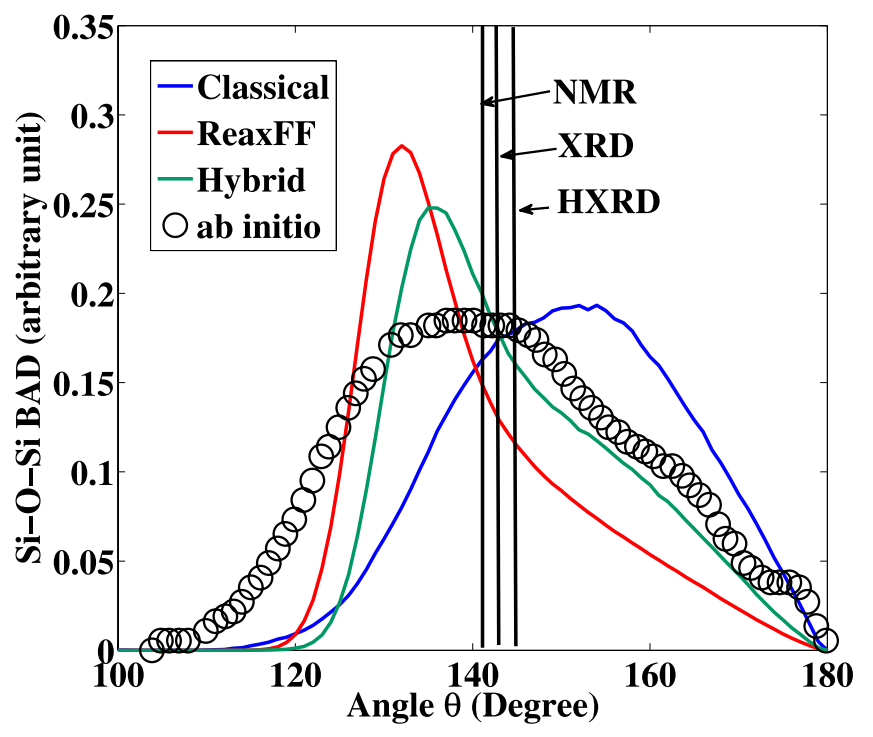

FIG. 6. Computed $\mathrm{Si}-\mathrm{O}-\mathrm{Si}$ bond angle distributions obtained by the three methods and from ab initio simulation $^{48}$. In each case, the average value of the distribution is indicated and compared to experimental values obtained from $\mathrm{NMR}^{44}, \mathrm{HXRD}^{43}$ and $\mathrm{XRD}^{45}$, represented by solid vertical lines.

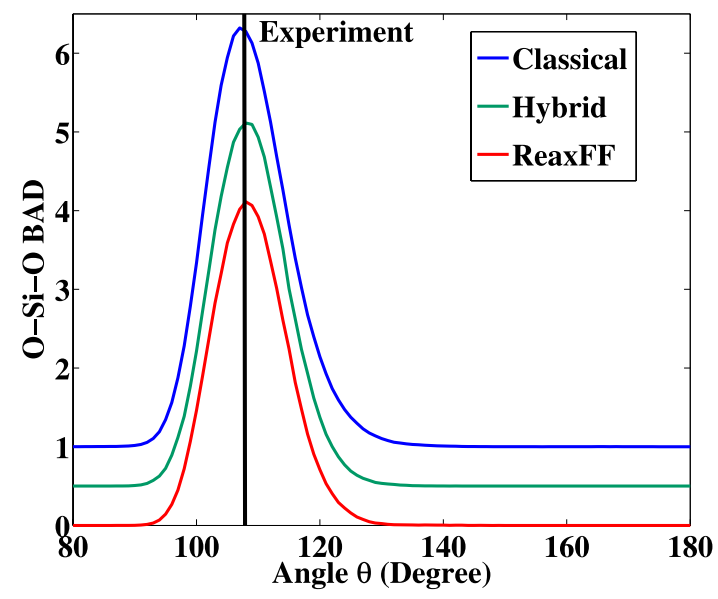

FIG. 7. Computed O-Si-O B bond angle distributions obtained for the three methods, compared with the experimental value of the average angle, represented by a solid vertical line ${ }^{36}$.

\subsection{Elasticity}

Next, the mechanical properties of the obtained glasses are examined by calculating the Young's and shear moduli. Arguably, if the structure of a glass as captured, e.g., by the PDF is rather easy to reproduce, even for poorly parameterized potentials, the elastic properties, which depend on the curvature of the potential, are more challenging 
to predict. Indeed, the elastic moduli computed for the glasses obtained by the three methods (shown in Table V) exhibit substantial variations. First, we note that the BKS potential over-predicts by more than $20 \%$ the shear modulus and by more than $30 \%$ the Young's modulus. These values are in agreement with previous studies and constitute a known limitation of the potential ${ }^{49}$. On the other hand, we note that the ReaxFF potential offers an excellent agreement with experiments ${ }^{50}$. On the contrary, the hybrid method leads to values that are fairly consistent with those predicted by the BKS values and, thereby, in poor agreement with experiments. Nevertheless, the ability of ReaxFF to predict elastic properties in excellent agreement with experiments is all the more remarkable since ReaxFF has not been parameterized with respect to glassy silica.

TABLE V. Computed shear and Young's moduli of the glasses obtained by the three methods, compared with experimental data.

$$
\text { Classical Hybrid ReaxFF } \quad \text { Experiment }{ }^{\mathrm{a}}
$$

\begin{tabular}{lrrrr}
$\begin{array}{l}\text { Shear modulus } \\
(\mathrm{GPa})\end{array}$ & $40.1 \pm 1.5$ & $42.0 \pm 0.4$ & $32.2 \pm 0.8$ & 31.3 \\
Young's & & & & \\
modulus (GPa) & $101.5 \pm 2.9$ & $104.6 \pm 1.1$ & $80.4 \pm 1.9$ & 72.9 \\
\hline
\end{tabular}

\subsection{Self-diffusion}

Finally, we evaluate the ReaxFF's ability to simulate the dynamics of the supercooled glass-forming liquid, that is, at temperature larger than the glass transition temperature $\left(T_{\mathrm{g}}\right)$. To this end, we calculated the mean-square displacement (MSD) versus time of the $\mathrm{O}$ atoms and checked that a diffusive regime is obtained, which manifests by a slope of one in $\log -\log$ scale. The self-diffusion constants $D$ of the $\mathrm{O}$ atoms in the silica glass-forming liquid were then evaluated by fitting the MSDs with the Einstein equation in the diffusive regime. Note that, since the simulations are performed with equilibrated liquids above $T_{\mathrm{g}}$, the distinction between the full ReaxFF and hybrid methods is no longer relevant and, as such, the results reflect solely the properties of the respective force fields. 


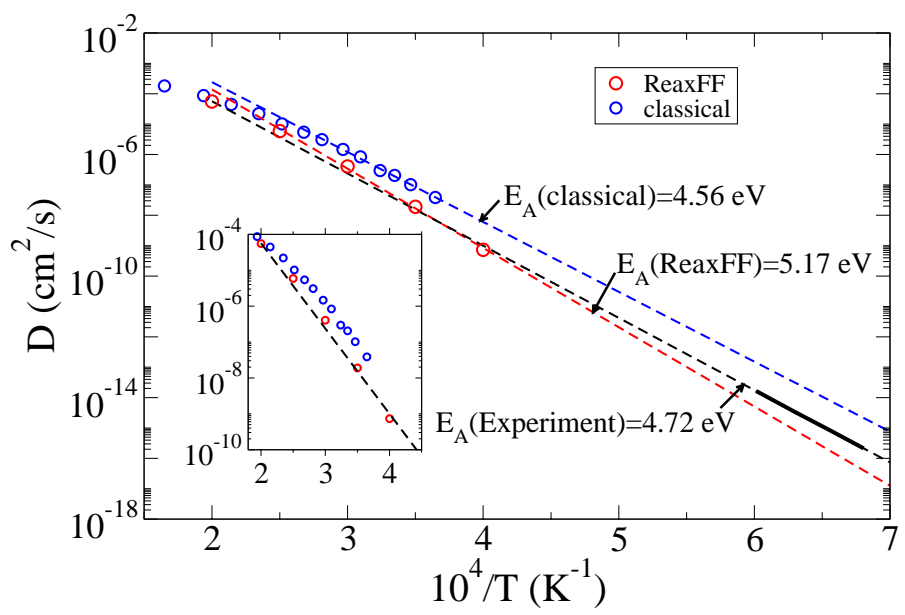

FIG. 8. Computed self-diffusion constants $D$ of the $\mathrm{O}$ atoms in the supercooled liquid state, using the BKS (blue circles) and ReaxFF (red circles) potentials. The values are compared with available experimental data (solid black line) ${ }^{51}$. For comparison, the simulated and experimental values are extrapolated (dashed lines) to higher and lower temperature, by assuming an Arrhenius-like behavior. The inset contains the same data, zoomed on the high temperature range.

Figure 8 shows the self-diffusion constants with respect to the inverse of the temperature $T$, for the ReaxFF and classical (BKS in this case) methods. An Arrhenius-like behavior, which is manifested by a linear trend in logarithm scale, can be clearly identified for both methods. This allows us to compare our results with the experimental data obtained at lower temperature by directly extrapolating the calculated values ${ }^{51}$. It is however important to point out that the simulated diffusion constants, especially those simulated with the BKS potential, eventually begin to show a non-linear behavior at higher temperatures. We therefore exclude these non-linear portions from the extrapolation.

As shown in Figure 8, a substantial difference in the magnitude of the diffusion constants is observed between the ReaxFF and BKS potentials. We observe that the diffusion constants predicted by the BKS potential are systematically about one order of magnitude higher than the extrapolated experimental data (see the insert of Figure 8). In contrast, ReaxFF offers a significantly better agreement in term of the magnitude. In addition, the activation energy, as calculated from the slope of the diffusion constants in logarithmic scale, is well reproduced by both ReaxFF and BKS. (see Figure 8).

\section{DISCUSSION}

In spite of decades of research, although glassy silica is the base structural unit for all silicate glasses, its structure remains an intriguing issue. As such, since they provide a direct access to the atomic configurations, MD simulations can bring critical insights and offer information otherwise not accessible from experiments. Overall, the 
structure predicted by ReaxFF offers a good agreement with available experimental data, both at the short- and medium-range order. Such an improvement is likely to arise from a more elaborated multi-body physical model and a better parameterization. For instance, it is not surprising that the ReaxFF potential leads to improved bond angle distributions, as it features three-body energy terms that constraint the angles.

As mentioned before, most classical inter-atomic potentials typically succeed in predicting a reasonable structure for glasses. Indeed, any rough parameterization would lead to the correct bond distances and coordination numbers. However, ReaxFF appears to offer significant enhancements for properties that are more sensitive to the details of the potential, like the elastic moduli or the diffusion constants. Clearly, further studies would be needed to better understand if these improvements arise from the specific features of ReaxFF (dependence of the potential on the local environment, ability to break and form bonds, and use of non-constant charges), or if a better parameterized conventional classical potential would offer the same level of performance. However, it can reasonably be assumed that the ability to break and form bonds plays a critical role to produce a realistic mechanism for the diffusion. As the atomic diffusion in silicate supercooled liquids is believed to occur through a hoping mechanism ${ }^{52,53}$ involving the creation of over- or under-coordinated intermediate species, the dependence of the potential on the local environment of the atoms is also likely to explain the better prediction of the magnitude of the self-diffusion constants by ReaxFF. This is also encouraging in the sense that an improved description of the dynamics of the supercooled liquid should ensure a more realistic cooling process and, as a result, a more reasonable structure in the final glassy state.

We new critically evaluate the outcome of the hybrid method tested in this study. Although similar methods have been adopted before (e.g., for ab initio or DFT simulations of glasses ${ }^{54,55}$ ), we observe that, in the case of silica, it produces a structure featuring mixed features arising both from the classical and ReaxFF potentials, rendering it less desirable. We therefore do not recommend using such a hybrid method for silica. Indeed, the hybrid method appears to preserve, to some degree, the medium-range order obtained with the classical method (as evidenced by the position of the FSDP and $\mathrm{Si}-\mathrm{Si} \mathrm{PDF} / \mathrm{Si}-\mathrm{O}-\mathrm{Si}$ BAD shoulder), while adopting the short-range order predicted by the ReaxFF potential. This is not surprising as, although local relaxation events (e.g., variations of the average angle) are possible after switching from the BKS to ReaxFF potential, larger range relaxation is not likely to occur. In fact, any variations of the medium range order of the glass, e.g., by changing the ring statistics, would require breaking and reforming bonds, which would be very unfavorable at room temperature and, indeed, is 
not observed in the present simulations. As such, the hybrid method can be problematic for the properties that do not only depend on the short-range order. As a matter of fact, as indeed observed here, the Young's and shear moduli predicted by the hybrid method show a poor agreement with experiments. Once again, this is not surprising as, although the elastic moduli strongly depend on the stiffness of the individual bonds and angles, they are also sensitive to the medium-range topology of the glassy network. Clearly, merely restoring the structure and interactions at the short-range, without a proper medium-range order, is not sufficient to predict realistic material properties. At the same time, it is worth noting that, for glasses featuring a less rigid network (e.g., alkali or alkaline earth silicates), the hybrid method may perform better, as the more open glass structure will permit a higher degree of relaxation. This will be tested in future studies, as using such a hybrid method, which comes with a lower computational cost than simulations relying fully on ReaxFF, would constitute an attractive option.

This study also highlights the fact that, to assess the quality of an inter-atomic potential, it is required to look beyond the usual structure characterizations (PDF and structure factor) and to perform an in-depth characterization of the environment of each element, as well as to compute more advanced properties like the elasticity. In order to facilitate further investigations within the community, the data file of the silica structure generated using the ReaxFF potential, in the format of LAMMPS package data file, has been uploaded to "openKIM" w5 with id RD_965415303158_000. “openKIM” is an online open source tool for molecular dynamics simulations of materials.

\section{CONCLUSIONS}

We conducted thorough tests of the ReaxFF reactive potential in simulating glassy silica, the foundational material for silicate glasses. We show that ReaxFF can produce a realistic glassy silica structure. Especially, we find ReaxFF to offer an improved level of agreement with experimental data at the medium-range order with respect to the well-established BKS potential. ReaxFF also provides substantial enhancements in the prediction of the elastic properties of the glass and of the dynamical properties of the supercooled liquid. Overall, ReaxFF provides an improved accuracy with respect to conventional classical inter-atomic potentials, while retaining higher computational efficiency than ab initio methods. Thanks to its ability to handle different atomic local environments, ReaxFF appears as a promising option for glass simulations, especially when more elements become available and thoroughly evaluated. In addition, its ability to model the formation and breaking of bonds, as observed during chemical reactions, would allow to study the surface reactivity of silicate glasses, which, due to intrinsic limitations, 
would be impossible through conventional classical molecular dynamics simulations, and, due to computational limitations, would be challenging through $a b$ initio methods.

\section{ACKNOWLEDGEMENTS}

The authors acknowledge partial financial support for this research provided by the University of California, Los Angeles (UCLA) and by the National Science Foundation (CAREER Award: 1235269). Access to computational resources was provisioned by the Physics of AmoRphous and Inorganic Solids Laboratory at UCLA. The contents of this paper reflect the views and opinions of the authors, who are responsible for the accuracy of the datasets presented herein, and do not reflect the views and/or policies of the funding agencies, nor do the contents constitute a specification, standard or regulation. 


\section{REFERENCES}

1. van Duin, A. C. T. et al. ReaxFFSiO Reactive Force Field for Silicon and Silicon Oxide Systems. J. Phys. Chem. A 107, 3803-3811 (2003).

2. Tersoff, J. Empirical interatomic potential for silicon with improved elastic properties. Phys. Rev. B 38, 9902-9905 (1988).

3. Brenner, D. W. Empirical potential for hydrocarbons for use in simulating the chemical vapor deposition of diamond films. Phys. Rev. B 42, 9458-9471 (1990).

4. Kim, K. et al. Ripping Graphene: Preferred Directions. Nano Lett. 12, $293-297$ (2012).

5. Jensen, B. D., Bandyopadhyay, A., Wise, K. E. \& Odegard, G. M. Parametric Study of ReaxFF Simulation Parameters for Molecular Dynamics Modeling of Reactive Carbon Gases. J. Chem. Theory Comput. 8, 3003-3008 (2012).

6. Mueller, J. E., van Duin, A. C. T. \& Goddard, W. A. Development and Validation of ReaxFF Reactive Force Field for Hydrocarbon Chemistry Catalyzed by Nickel. J. Phys. Chem. C 114, 4939-4949 (2010).

7. Norman, P. et al. The Structure of Silica Surfaces Exposed to Atomic Oxygen. J. Phys. Chem. C 117, 9311-9321 (2013).

8. Kim, S.-Y. et al. Development of a ReaxFF Reactive Force Field for Titanium Dioxide/Water Systems. Langmuir 29, 7838-7846 (2013).

9. Raju, M., Kim, S.-Y., van Duin, A. C. T. \& Fichthorn, K. A. ReaxFF Reactive Force Field Study of the Dissociation of Water on Titania Surfaces. J. Phys. Chem. C 117, 10558-10572 (2013).

10. Zhang, Q. et al. Adhesion and nonwetting-wetting transition in the $\$ \backslash$ mathrm $\{\mathrm{Al}\} /$ ensuremath $\{\backslash$ alpha $\}$ $\{\backslash \text { mathrm }\{\mathrm{Al}\}\}_{-}\{2\}\{\backslash \text { mathrm }\{\mathrm{O}\}\}_{-}\{3\} \$$ interface. Phys. Rev. B 69, 045423 (2004).

11. Abdolhosseini Qomi, M. J. et al. Combinatorial molecular optimization of cement hydrates. Nat. Commun. 5, (2014).

12. Bauchy, M., Abdolhosseini Qomi, M. J., Bichara, C., Ulm, F.-J. \& Pellenq, R. J.-M. Nanoscale Structure of Cement: Viewpoint of Rigidity Theory. J. Phys. Chem. C 118, 12485-12493 (2014).

13. Bauchy, M., Qomi, M. J. A., Ulm, F.-J. \& Pellenq, R. J.-M. Order and disorder in calcium-silicate-hydrate. J. Chem. Phys. 140, 214503 (2014).

14. Bauchy, M. et al. Fracture toughness of calcium-silicate-hydrate from molecular dynamics simulations. $J$. Non-Cryst. Solids 419, 58-64 (2015).

15. Theofanis, P. L., Jaramillo-Botero, A., Goddard, W. A. \& Xiao, H. Nonadiabatic Study of Dynamic Electronic Effects during Brittle Fracture of Silicon. Phys. Rev. Lett. 108, 045501 (2012).

16. Buehler, M. J., Tang, H., van Duin, A. C. T. \& Goddard, W. A. Threshold Crack Speed Controls Dynamical Fracture of Silicon Single Crystals. Phys. Rev. Lett. 99, 165502 (2007).

17. Bauchy, M., Qomi, M. J. A., Bichara, C., Ulm, F.-J. \& Pellenq, R. J.-M. Rigidity Transition in Materials: Hardness is Driven by Weak Atomic Constraints. Phys. Rev. Lett. 114, 125502 (2015).

18. Fogarty, J. C., Aktulga, H. M., Grama, A. Y., Duin, A. C. T. van \& Pandit, S. A. A reactive molecular dynamics simulation of the silica-water interface. J. Chem. Phys. 132, 174704 (2010).

19. Rimsza, J. M., Deng, L. \& Du, J. Molecular dynamics simulations of nanoporous organosilicate glasses using Reactive Force Field (ReaxFF). J. Non-Cryst. Solids doi:10.1016/j.jnoncrysol.2015.04.031

20. Bauchy, M., Guillot, B., Micoulaut, M. \& Sator, N. Viscosity and viscosity anomalies of model silicates and magmas: A numerical investigation. Chem. Geol. 346, 47-56 (2013).

21. van Duin, A. C. T., Dasgupta, S., Lorant, F. \& Goddard, W. A. ReaxFF: A Reactive Force Field for Hydrocarbons. J. Phys. Chem. A 105, 9396-9409 (2001).

22. Rappe, A. K. \& Goddard, W. A. Charge equilibration for molecular dynamics simulations. J. Phys. Chem. 95, 3358-3363 (1991).

23. Demiralp, E., Çağin, T. \& Goddard, W. A. Morse Stretch Potential Charge Equilibrium Force Field for Ceramics: Application to the Quartz-Stishovite Phase Transition and to Silica Glass. Phys. Rev. Lett. 82, 1708-1711 (1999).

24. Kieu, L.-H., Delaye, J.-M., Cormier, L. \& Stolz, C. Development of empirical potentials for sodium borosilicate glass systems. J. Non-Cryst. Solids 357, 3313-3321 (2011).

25. Bauchy, M. Structural, vibrational, and elastic properties of a calcium aluminosilicate glass from molecular dynamics simulations: The role of the potential. J. Chem. Phys. 141, 024507 (2014). 
26. Plimpton, S. Fast Parallel Algorithms for Short-Range Molecular Dynamics. J. Comput. Phys. 117, 1-19 (1995).

27. Vollmayr, K., Kob, W. \& Binder, K. Cooling-rate effects in amorphous silica: A computer-simulation study. Phys. Rev. B 54, 15808-15827 (1996).

28. Nakano, A., Kalia, R. K. \& Vashishta, P. First sharp diffraction peak and intermediate-range order in amorphous silica: finite-size effects in molecular dynamics simulations. J. Non-Cryst. Solids 171, 157-163 (1994).

29. Tangney, P. \& Scandolo, S. An ab initio parametrized interatomic force field for silica. J. Chem. Phys. 117, 8898-8904 (2002).

30. Yuan, F. \& Huang, L. Molecular dynamics simulation of amorphous silica under uniaxial tension: From bulk to nanowire. J. Non-Cryst. Solids 358, 3481-3487 (2012).

31. Roder, A., Kob, W. \& Binder, K. Structure and dynamics of amorphous silica surfaces. J. Chem. Phys. 114, 7602-7614 (2001).

32. Yuan, F. \& Huang, L. Brittle to Ductile Transition in Densified Silica Glass. Sci. Rep. 4, (2014).

33. Bauchy, M. Structural, vibrational, and elastic properties of a calcium aluminosilicate glass from molecular dynamics simulations: The role of the potential. J. Chem. Phys. 141, 024507 (2014).

34. Pedone, A., Malavasi, G., Cormack, A. N., Segre, U. \& Menziani, M. C. Insight into Elastic Properties of Binary Alkali Silicate Glasses; Prediction and Interpretation through Atomistic Simulation Techniques. Chem. Mater. 19, 3144-3154 (2007).

35. Lane, J. M. D. Cooling rate and stress relaxation in silica melts and glasses via microsecond molecular dynamics. Phys. Rev. E 92, 012320 (2015).

36. Grimley, D. I., Wright, A. C. \& Sinclair, R. N. Neutron scattering from vitreous silica IV. Time-of-flight diffraction. J. Non-Cryst. Solids 119, 49-64 (1990).

37. Wright, A. C. Neutron and X-ray amorphography. J. Non-Cryst. Solids 106, 1-16 (1988).

38. Lorch, E. Neutron diffraction by germania, silica and radiation-damaged silica glasses. J. Phys. C Solid State Phys. 2, 229 (1969).

39. Mazurin, O. V., Streltsina, M. V. \& Shvaiko-Shvaikovskaya, T. P. Handbook of glass data, Part A. Silica Glass Bin. Silic. Glas. (1983).

40. Pilla, O., Angelani, L., Fontana, A., Gonçalves, J. R. \& Ruocco, G. Structural and dynamical consequences of density variation in vitreous silica. J. Phys. Condens. Matter 15, S995 (2003).

41. Wright, A. C. The comparison of molecular dynamics simulations with diffraction experiments. J. NonCryst. Solids 159, 264-268 (1993).

42. Henderson, G. S. A Si K-edge EXAFS/XANES study of sodium silicate glasses. J. Non-Cryst. Solids 183, 43-50 (1995).

43. Poulsen, H. HF Poulsen, J. Neuefeind, H.-B. Neumann, JR Schneider, MD Zeidler. J Non-Cryst Solids 188, 63 (1995).

44. Pettifer, R. F., Dupree, R., Farnan, I. \& Sternberg, U. NMR determinations of Si $\square \mathrm{O} \square$ Si bond angle distributions in silica. J. Non-Cryst. Solids 106, 408-412 (1988).

45. Mozzi, R. L. \& Warren, B. E. The structure of vitreous silica. J. Appl. Crystallogr. 2, 164-172 (1969).

46. Mauri, F., Pasquarello, A., Pfrommer, B. G., Yoon, Y.-G. \& Louie, S. G. Si-O-Si bond-angle distribution in vitreous silica from first-principles $\$\{\}^{\wedge}\{29\} \backslash$ mathrm $\{\mathrm{Si}\} \$$ NMR analysis. Phys. Rev. B 62, R4786-R4789 (2000).

47. O'Keeffe, M. \& Hyde, B. G. On Si-O-Si configurations in silicates. Acta Crystallogr. Sect. B 34, 27-32 (1978).

48. Van Ginhoven, R. M., Jónsson, H. \& Corrales, L. R. Silica glass structure generation for Itextit $\{a b$ initio calculations using small samples of amorphous silica. Phys. Rev. B 71, 024208 (2005).

49. Muralidharan, K., Simmons, J. H., Deymier, P. A. \& Runge, K. Molecular dynamics studies of brittle fracture in vitreous silica: Review and recent progress. J. Non-Cryst. Solids 351, 1532-1542 (2005).

50. Bansal, N. P. \& Doremus, R. H. Handbook of Glass Properties. (Elsevier, 2013).

51. Jr, J. C. M. Self-diffusivity of network oxygen in vitreous SiO2. Appl. Phys. Lett. 45, 1187-1189 (1984).

52. Bauchy, M. \& Micoulaut, M. From pockets to channels: Density-controlled diffusion in sodium silicates. Phys. Rev. B 83, 184118 (2011).

53. Bauchy, M., Guillot, B., Micoulaut, M. \& Sator, N. Viscosity and viscosity anomalies of model silicates and magmas: A numerical investigation. Chem. Geol. 346, 47-56 (2013).

54. Koudriachova, M. V., Beckers, J. V. L. \& de Leeuw, S. W. Computer simulation of the quartz surface: a combined ab initio and empirical potential approach. Comput. Mater. Sci. 20, 381-386 (2001).

55. Benoit, M., Ispas, S., Jund, P. \& Jullien, R. Model of silica glass from combined classical and ab initio molecular-dynamics simulations. Eur. Phys. J. B - Condens. Matter Complex Syst. 13, 631-636 (2000). 
56. Tadmor, E. B., Elliott, R. S., Sethna, J. P., Miller, R. E. \& Becker, C. A. Knowledgebase of Interatomic Models (KIM). (2011). at <https://openkim.org> 